and age. But, simple as this sounds, it has proved extremely difficult in practice, and only recently have there been signs of success.

The present volume contains 23 excellent articles which range over all these topics. A number give details of techniques; many of them are valuable reviews of the work which has followed from the advances in technique.

It is seldom that a composite volume maintains such a uniformity of aim and standard, and the editor is to be warmly congratulated. One may hope that his labours will give fresh encouragement to an important and rapidly growing branch of biology.

Michael SwanN

\section{ADVANCES IN FORESTRY}

\section{International Review of Forestry Research}

Vol. 1. Edited by John A. Romberger and Peitsa Mikola. Pp. xi +404 . (New York: Academic Press, Inc.; London: Academic Press, Inc. (London), Ltd., 1964.) $93 s$.

7 HE appearance of Volume 1 of International Review 1 of Forestry Research, a series in which it is proposed to provide authoritative review articles covering, internationally, all the important publications in particular fields of forestry knowledge, is very welcome. It is well produced, printed and bound, though for its size it is expensive, even according to modern standards. The stated aim is to provide an international publication medium in which knowledge and ideas of forest scientists will be "collected, evaluated and synthesized" within definite fields of work.

Hitherto, the only source of such synthesis of knowledge on a truly international scale has been the "Review Articles" that appear at the beginning of each number of Forestry Abstracts. These are of a uniformly high standard of presentation and completeness-factual, authoritative and often tough reading. As it is only possible to issue three or four of these a year, the rate of coverage of the wide field of forest science by them is undesirably slow, and any additional publication provid. ing such reviews is valuable.

The compilation of these review articles is an exceedingly laborious task if it is to be done properly, and it is difficult to find active research workers who will give up the time to do it. Yet the reviews must be done by experts who are thoroughly familiar with their subjects. Also, if a uniformly high standard is to be achieved, editing has to be very strict. In the volume under discussion seven subjects are reviewed and the quality and completeness of the articles are variable. Very high standards are achieved in four of them. Leo Heikuranen of Finland presents an excellent account of the "Improvement of Forest Growth on Poorly Drained Peat Soils", covering work mainly in Finland, Britain and North America, and giving about 250 references to literature. It is a curious coincidence that, almost simultaneously, M. S. Botch has covered a good deal of the same ground in a comprehensive review article (in Russian) entitled "Principal Problems and Trends in the Development of Peatland Science in European Countries during the Period 1945-63" in the Russian Botanical Journal of February 1965. This covers work in all European countries (except Russia, and also Spain, Portugal and Greece for which no data were available). Had each had access to theother's bibliography, the coverage would have been notably improved. The article by Carl Olaf Tamm on "The Determination of Nutrient Requirements of Forest Stands" (about 220 references), and that by Ken-Ichi Hatamo and Sumikiko Asakawa on "Physiological Processes in Forest Tree Seeds during Maturation, Storage and Germination" (about 280 references), are models of their kind, being critical and sustaining the interest of the reader by clear concise writing. Lalit M. Srivastava's article on "Anatomy, Chemistry and Physiology of Bark" (some 240 references) also sets a high standard, though it is written in much more technical language and is only readable by an expert wood anatomist who is also a biochemist; but this is probably inevitable in a subject of this nature.

The other three articles are more variable in quality and more discursive in mode of presentation. Kurt Mantel's "History of the International Science of Forestry with special Consideration of Central Europe" is, in spite of its title, restricted to Germany and France and is an annotated list of references to publications by the early foresters in those countries in various defined fields of forestry. While mentioning many famous figures, little information is given about the contributions they made to forest science. It is thus a guide to reading rather than a synthesis of knowledge; and, as such, has its use. The review by Charles W. Ralston on "Evaluation of Forest Site Productivity" deals with a vague subject in a rather indefinite way. It could have been more critical and is too tolerant of the mystique behind much of the work carried out in this field. It omits much important work carried out in Europe and does not mention the ideas of Czarnowski in his own country. Nevertheless it provides many useful references for anyone studying this field. Finally, A. D. Voûte gives a rather general article on "Harmonious Control of Forest Insects", a useful summary of ideas on the various controlling agencies, natural and artificial, and how they might be combined to maintain populations at tolerably low levels. No very clear recommendations emerge. It has a useful bibliography (180 references), and is good reading for students.

All in all, this series has got off to a very promising start. It is to be hoped that the standards of the best of the articles will become general and, above all, that the coverage will be fully international, not omitting tropical forest science. It is important that when an article covers work from a limited geographical area, this is clearly stated in the title. The value of the articles is greatly enhanced when the reviews, as in several cases in this volume, include sources of literature that are inaccessible to the average reader. This may mean calling in translators to deal with unfamiliar languages. Finally, all forest scientists and students should be eternally grateful to those who undertake these review articles, as their compilation is a very laborious and often thankless task.

M. V. LAURIE

\section{BIRDS' EGG SHELLS}

Wilhelm von Nathusius, 1821-1899, on Avian Eggshells A Translated and Edited Version of his Work. By Prof. Cyril Tyler. Pp. $104+18$ plates. (Reading: C. Tyler. Obtainable from the Berkshire Printing Co., Ltd., Oxford Road, Reading.) 43s. $6 d$.

URING 1964 the British Egg Marketing Board was forced to exclude from sales about $1.2 \times 10^{6}$ cases of eggs because of shell defects. Each case contained 30 dozen eggs so that the total number having fragile or cracked shells was approximately $3.6 \times 10^{7}$ and represented a financial loss of about three million pounds. The modern laying hen has been bred to produce nearly three hundred eggs in a year, but little or no attention has been paid to the fact that these hens do not appear to have paralleled their ovulation rate with a concomitant improvement in calcium absorption from the gut. Moreover, some hens persist in laying many eggs with defective shells; this may be an inherited trait.

The deposition of the inorganic components on or around the organic matrix of the egg shell is by no means understood; Prof. Tyler has devoted much time to the study of eggshell-strength, but as yet we have no really satisfactory method to test against the mechanical illtreatment the hen's egg receives between cloaca and customer, nor can we readily detect hidden defects which 